\title{
Ascites due to ovarian hyperstimulation syndrome: A case report and review of literature
}

\author{
Charles Panackel, Baiju, T. R. Radha, George Cherian
}

Objectives: To report a case of ovarian hyperstimulation syndrome (OHSS), to discuss the differential
diagnosis and to give a review of current evidences in diagnosis and management of ovarian hyperstimulation
syndrome. Design: A detailed description of a case of OHSS followed by a thorough review of case reports,
randomized controlled trials and review articles to assess the current modalities of diagnosis and management
of ovarian hyperstimulation syndrome. Results: A 29 -year-old female was admitted with dyspnoea and features
suggestive of polyserositis. Patient was evaluated for causes of polyserositis. She gave history of ovulation
induction and invitro fertilization one week prior to the onset of dyspnoea. With the positive history of invitro
fertilization and negative markers for other causes of capillary leak syndrome the patient was diagnosed to
have OHSS. She was treated and improved within a week. Conclusion: Diagnosis and management of
ovarian hyperstimulation syndrome requires high index of suspicion, prompt investigation and early initiation
of effective therapy. With correct diagnosis and early treatment, most patients recover.
Key words: Ascites, fertilization, ovarian hyperstimulation syndrome, ovarian, pleural effusion

\section{Introduction}

Ovarian hyperstimulation syndrome (OHSS) is a rare complication of ovulation induction therapy. The syndrome occurs in the luteal phase or during early part of pregnancy. The syndrome was first described in 1941 and first fatal case of OHSS with renal failure and death was described in 1951. It can occur following ovarian induction with any agent. Rare cases of OHSS have been described at the beginning of spontaneous pregnancy due to mutations in follicle stimulating hormone receptor. The symptoms can range from nausea, vomiting and mild abdominal discomfort to severe disease with ascites, hydrothorax and renal failure. Treatment is mainly supportive.

\section{From:}

Department of Medicine, Medical College, Kottayam, Kerala, India

\section{Correspondence:}

Dr. Charles Panackel, 10 A 2 Heera Kinara Classic, Kesavadasapuram,

Trivandrum, India. E-mail: charlespanackel@hotmail.com

\section{Case Report}

A 29-year-old female presented to us with history of nausea and vomiting of one-week duration followed by progressive abdominal distension and dyspnoea of three days. She had no chest pain orthopnea or paroxysmal nocturnal dyspnoea. There was no fever, cough, hemoptysis or weight loss. She did not have jaundice, altered sleep rhythm or bleeding diathesis. Patient did not have any frothing of urine, oliguria or hematuria.

She was apparently normal till one week back when she developed nausea and vomiting. She did not give a history of pulmonary tuberculosis. There was no past history of arthritis oral ulcers or skin rash. Patient was married for five years and gave history of infertility treatment for last two years. She gave history of invitro fertilization two weeks earlier in another hospital.

On examination patient was breathless, her pulse 
rate was 112 per minute, blood pressure was 110/70 $\mathrm{mm}$ of $\mathrm{Hg}$ in right upper limb. Jugulars were not distended. General examination was normal. Abdominal examination revealed the presence of ascites. There was no hepatosplenomegaly or palpable mass. Respiratory system examination revealed moderate right pleural effusion. Other systems were normal.

Laboratory investigations revealed a hemoglobin of $14 \mathrm{gm} \%$, total count 7320, ESR $16 \mathrm{~mm}$ in the first hour, platelet count and peripheral smear were normal, blood urea $14 \mathrm{mg} \%$, serum creatinine $0.6 \mathrm{mg} \%$, S. bilirubin 0.8 $\mathrm{mg} \%$, ALT $18 \mathrm{IU} / \mathrm{L}$, AST $16 \mathrm{IU} / \mathrm{L}$, alkaline phosphatase $56 \mathrm{IU} / \mathrm{L}$, total protein $5.4 \mathrm{gm} \%$, serum albumin $3.0 \mathrm{gm} \%$, serum sodium $142 \mathrm{meq} / \mathrm{L}$, serum potassium $4.6 \mathrm{meq} / \mathrm{L}$, pO2 $126 \mathrm{mmHg}, \mathrm{pCO} 234 \mathrm{mmHg}, \mathrm{pH} 7.44$. Serum amylase and lipase were normal. Chest $\mathrm{X}$-ray revealed moderate pleural effusion and Electrocardiogram showed sinus tachycardia without any ST-T abnormalities. A transabdominal and transvaginal sonogram was done which showed moderate ascites with right pleural effusion. Liver, spleen and kidneys were normal; there were no lymph nodes. Uterus did not reveal a gestational sac and both ovaries showed multiple small cysts. In view of invitro fertilization, a pregnancy test was done and was negative. Pleural fluid and ascitic fluid study are shown in Table 1.

So we had a young lady presenting with serous cavity effusion. She was evaluated for various causes of capillary leak syndrome. Antibody tests for Dengue, Leptospira, Human immunodeficiency virus and Epstein Barr virus were negative. Mantoux, antinuclear antibody, anti double stranded DNA antibody were negative. Her blood, urine and fluid cultures were sterile. The Thyroid function tests were normal. She did not give any history of insect or reptile bite. In view of history of invitro fertilization a diagnosis of ovarian hyperstimulation syndrome was made. Patient was admitted to ICU and treated with

\begin{tabular}{ll}
\hline Table 1: Results of pleural and ascitic fluid study \\
\hline Pleural fluid & Ascitic fluid \\
Protein $-3.8 \mathrm{gm} / \mathrm{dl}$ & Protein $-3.8 \mathrm{gm} / \mathrm{dl}$ \\
Albumin $-2.6 \mathrm{gm} / \mathrm{dl}$ & Albumin $-2.6 \mathrm{gm} / \mathrm{dl}$ \\
Sugar $-68 \mathrm{mg} \%$ & Sugar $-68 \mathrm{mg} \%$ \\
Count $-4 \mathrm{cells} / \mathrm{ml}$ & Count $-50 \mathrm{cells} / \mathrm{ml}$ predominant \\
all lymphocytes & mesothelial cells \\
Cytology negative for malignant & Cytology negative for malignant \\
cells & cells \\
AFB culture - Negative & AFB culture - Negative \\
ADA - 14 & ADA - 20 \\
\hline
\end{tabular}

human albumin infusion with close monitoring of vitals, urine output, renal function and electrolytes. She made a gradual recovery over the next ten days and was discharged.

\section{Discussion}

Ovarian hyperstimulation syndrome [OHSS] is an iatrogenic complication associated with invitro fertilization [IVF]. The syndrome is typically associated with exogenous gonadotrophin stimulation. It is also rarely seen with other agents like clomiphene citrate and gonadotrophin releasing hormones [ $\mathrm{GnRH}$ ]. Spontaneous occurrence of OHSS has been reported in rare instances during pregnancy. ${ }^{[1]}$ Familial cases of recurrent OHSS are seen in persons with mutations in Follicle Stimulating Hormone [FSH] receptors. ${ }^{[2,3]}$

\section{Etiopathogenesis}

Invitro fertilization techniques use $\mathrm{GnRH}$ agonists or antagonists and gonadotrophins to stimulate the ovary. Following stimulation, human chorionic gonadotrophin [HCG] is used to initiate ovulation and maintain luteal phase. In rare instances the ovarian stimulation can lead to excessive ovarian response. It is characterized by ovarian enlargement accompanied by over production of ovarian hormones and a host of ovarian vasoactive agents [Table 2]. These agents alone or together produce a state of increased capillary permeability. ${ }^{[4]}$

The hallmark of OHSS is an increased capillary permeability resulting in fluid shift from intravascular space to third space. HCG either endogenous (pregnancy derived) or exogenous plays a central role in pathogenesis of OHSS. HCG induces the release of vasoactive mediators, especially vascular endothelial growth factor [VEGF]. ${ }^{[5,6]}$ VEGF is the main angiogenic substance that leads to increase in capillary permeability. The serum

Table 2: Ovarian vasoactive agents implicated in ovarian hyperstimulation syndrome

Human chorionic gonadotropin
Vascular endothelial cell growth factor
Estradiol
Ovarian renin-angiotensin system
Von Willebrand factor
Cytokines
Prostaglandins
Insulin endothelial-cell adhesion molecules
Angiotensin
Histamines
Endothelin-1
Ovarian kinin-kallikrein system Interleukin-6


VEGF levels correlates with severity of OHSS. ${ }^{[5,6]}$ Ovarian renin-angiotensin also plays a role in maintaining OHSS. Plasma renin activity also correlates with severity of OHSS. ${ }^{[7]}$

\section{Epidemiology and risk factors}

The prevalence of moderate to severe OHSS ranges from $1-10 \%$ in major IVF programs. ${ }^{[8]}$ It is seen in approximately $2 \%$ of all IVF cycles. ${ }^{[9]}$ There are many risk factors associated with development of OHSS [Table 3]. ${ }^{[10]}$ The incidence of OHSS is related to the stimulation regime used. A high incidence is seen with the use of gonadotrophins. There is no difference whether urinary or recombinant gonadotrophins are used. ${ }^{[11]}$ Moderate OHSS is seen in $8 \%$ of stimulation cycles using clomiphene citrate. ${ }^{[10]}$ The data regarding use of GnRH agonist and antagonist are contradictory. ${ }^{[12]}$ The risk of OHSS is increased in women who experience a rapid rise in serum estradiol levels or in whom estradiol levels are more than $2500 \mathrm{pg} / \mathrm{ml}^{.{ }^{[13]}}$ Risk also increases with the number of developing follicles and the number of oocytes [ $>14$ ] retrieved during artificial reproduction technique $[\mathrm{ART}]^{\left[{ }^{[14]}\right.}$ Use of higher and repeated doses of HCG increases the risk of OHSS. Pregnancy not only increases the likelihood of OHSS, but also prolongs the duration and severity of symptoms. A higher incidence of OHSS is seen in patients with ultrasound evidence of polycystic ovarian syndrome [PCOS]. ${ }^{[15]}$

\section{Clinical features}

The hallmark of OHSS is shift of fluid from the intravascular to extra vascular space. ${ }^{[16]}$ Symptoms usually begin with a sensation of bloating, abdominal discomfort, nausea, vomiting and diarrhea. As the disease progress accumulation of fluid in the third space leads to ascites, pleural and pericardial effusion, oliguria, hemoconcentration, hypovolemia and electrolyte

\footnotetext{
Table 3: Risk factors for ovarian hyperstimulation syndrome

Young age (<35 years)

Low body mass index - asthenic habitus

Polycystic ovary syndrome

History of atopy or allergies

High serum estradiol

Previous episode of ovarian hyperstimulation syndrome

Multiple follicles

Higher or repeated doses of exogenous human

Chorionic gonadotropin

Gonadotropin-releasing hormone agonist protocol

Pregnancy
}

imbalance. ${ }^{[17-19]}$ Patient may present with acute abdominal pain due to torsion of adnexa.

Physical examination may reveal weight gain, increased abdominal girth, oliguria and signs of hypovolemia. Abdominal examination shows the presence of ascites. The ovaries may rarely be palpable. Presence of severe pallor should raise the suspicion of ovarian rupture and hemoperitoneum. One should always be on the look out for pleural and pericardial effusion, thromboembolism and adult respiratory distress syndrome (ARDS). These should be picked up early and managed to decrease morbidity and mortality.

Laboratory data is characterized by hemoconcentration (Hct $>45 \%$ ) and raised white blood cell and platelet counts. ${ }^{[19]}$ Electrolyte disturbances in the form of hyponatremia and hyperkalemia may be seen. Abnormal liver function tests are seen in $30 \%$ of patients with OHSS. ${ }^{20]}$ Hypoalbuminemia is universal. Raised serum creatinine points to severe OHSS. Low levels of $\lg A$ and $\lg G$ are seen in patients with severe OHSS and predisposes to infections. ${ }^{[21]}$ The plasma levels of renin, aldosterone, noradrenaline, antidiuretic hormone $[A D H]$ and atrial natriuretic peptide [ANP] are increased. ${ }^{[19,21]}$ Urinary sodium concentration is low in most patients. Ascitic fluid study reveals high protein and low cell counts. ${ }^{[1]}$

\section{Complication}

Pulmonary complications of OHSS include hydrothorax, pulmonary embolism, ARDS, pulmonary edema, atelectasis and intra alveolar hemorrhage. ${ }^{[16]}$ Pleural effusion develops in approximately $20 \%$ of severe OHSS. Pleural effusions are usually bilateral but can be unilateral and on the right. ${ }^{[16]}$ Thromboembolism is common in OHSS. High estrogen concentration, hemoconcentration, reduced circulating blood volume and thrombocytosis predispose the patient to thromboembolism. ${ }^{[22,23]}$ Elevated levels of procoagulants and fibrinolytic inhibitor agents have been reported in OHSS. Both arterial and venous thrombosis occurs. ${ }^{[24]}$ Venous thrombosis is more common in the upper limb and neck. Most arterial thrombi are intracerebral. ${ }^{[22,23]}$

Infections are increased in patients with OHSS. High incidence of infections is attributed to decreased lgG and IgA levels seen in OHSS. ${ }^{[25]}$ In a large series the incidence of febrile morbidity was $82.3 \% .{ }^{[25]}$ Most common 
cause for fever was urinary tract infection seen in $20.5 \%$ patients. The most common causative organisms isolated Proteus Mirabilis, klebsiella Pneumonia, Pseudomonas Aeroginosa, E.coli, Morganella Morgani and Proteus Vulgaris. ${ }^{[25]}$ Other rare complications include adnexal torsion, ovarian cyst rupture and ovarian cyst hemorrhage.

\section{Classification}

Rabau et al. classified ovarian hyperstimulation syndrome into mild, moderate and severe [Table 4]. ${ }^{[26]}$ In 1992 Navot et al. added a new stage, critical or life threatening OHSS. ${ }^{[27]}$ Depending on the time of onset OHSS has been classified into early onset OHHS and late onset OHSS. Early onset OHSS occurs within three to seven days after the administration of ovulatory dose of HCG and is caused by excessive preovulatory ovarian response to stimulation. Late OHSS is caused by a pregnancy related increase in HCG level and occurs more than one week after the increase. Late onset OHSS is more severe than early onset OHSS. ${ }^{[28]}$

\section{Management}

There is no specific treatment for OHSS. Therapy is

\section{Table 4: Classification of ovarian hyperstimulation syndrome}

Mild ovarian hyperstimulation syndrome

Urinary estrogen $>150>\mathrm{g} / 24 \mathrm{~h}$

Urinary pregnanediol $>10 \mathrm{mg} / 24 \mathrm{~h}$

Enlargement of ovaries with or without palpable cyst formation

Moderate ovarian hyperstimulation syndrome

Abdominal distension, nausea

Urinary estrogen $>150>\mathrm{g} / 24 \mathrm{~h}$

Urinary pregnanediol $>10 \mathrm{mg} / 24 \mathrm{~h}$

Enlargement of ovaries with or without palpable cyst formation

Vomiting and diarrhea

Severe ovarian hyperstimulation syndrome

Variably enlarged ovary

Massive ascites with or without hydrothorax

Hematocrit N45\%

WBC count N15,000

Oliguria

Creatinine level $1.0-1.5 \mathrm{mg} / \mathrm{dL}$

Creatinine clearance $<60 \mathrm{~mL} / \mathrm{min}$

Liver dysfunction

Anasarca

Life-threatening ovarian hyperstimulation syndrome

Variably enlarged ovary

White blood cell count $>25,000$

Hematocrit $>55 \%$

Creatinine level $>1.6 \mathrm{mg} / \mathrm{dL}$

Reduction in creatinine clearance $<50 \mathrm{~mL} / \mathrm{min}$

Oliguria

Renal failure

Tense ascites with or without hydrothorax

Thromboembolic phenomena

Acute respiratory distress syndrome mainly supportive. The syndrome is self-limiting and resolution parallels the fall in HCG levels. Mild cases can be managed on outpatient basis with daily measurement of weight, urinary output, avoidance of strenuous activity and sexual intercourse. Oral fluid intake should be monitored. Patients should have serial measurements of hematocrit, electrolytes and creatinine..$^{[10,29,30]}$

Moderate OHSS usually subsides with bed rest for two to three weeks. They should be monitored closely. Serum beta HCG levels should be measured to confirm pregnancy. Pelvic examination should be avoided to decrease the risk of ovarian rupture..$^{[10,17]}$

Patients with severe OHSS should be managed as inpatient. Hemodynamic and respiratory status should be assessed. A thorough physical examination should be done to look for evidence of venous thrombosis. An intravenous line, ideally a subclavian catheter should be placed to measure central venous pressure [CVP]. Whether to put an indwelling urinary bladder catheter is controversial because of increased risk of urinary tract infection. ${ }^{[10,13]}$

All patients with severe OHSS should be monitored daily with hematocrit, total count, electrolytes, liver function tests, renal function tests. ${ }^{[10,13,31,32]}$ Ultrasound abdomen is done to measure the size of ovaries and to determine the presence of ascites. If the patient has dyspnoea, a chest radiograph after shielding the uterus should be obtained. Oxygen saturation and arterial blood gases should be done for all patients with dyspnoea. If respiratory status worsens or respiratory failure develops endotracheal intubation and mechanical ventilation may be required. When pericardial effusion is suspected an echocardiogram is obtained. ${ }^{[31,32]}$

Medical management is mainly to maintain circulatory function and prevent organ dysfunction. The intravascular volume should be maintained to prevent hemoconcentration and allow sufficient urine output. Initial fluid of choice is crystalloids. ${ }^{[10,30]}$ In patients with hematocrit more than $45 \%$ or hypoalbuminemia less than $30 \mathrm{gm} / \mathrm{L}$ or ascites, human albumin is the plasma expander of choice. Once sufficient volume expansion has been achieved and the hematocrit is less than $36 \%$ frusemide should be given to assess the renal function. Premature or overzealous use of diuretics may aggravate hypovolemia and hemoconcentration leading to renal 
dysfunction and thromboembolism. ${ }^{[10,13,30,35]}$ Intravascular volume expanders like fresh frozen plasma and dextran has no advantage over albumin. ${ }^{[32,35]}$ Intravascular volume expansion with dextran has been associated with development of ARDS in patients with OHSS. ${ }^{[33,35]}$

In patients with hydrothorax who are not symptomatic, conservative management is sufficient. If patient has respiratory symptoms thoracocentesis should be done. If ARDS develops patient should be ventilated with low tidal volume ( $6 \mathrm{ml} / \mathrm{kg}$ body weight) and plateau pressure less than $30 \mathrm{~cm}$ of water. Patient should be investigated for evidence of infection. Use of steroids in ARDS is not recommended at present. ${ }^{[34]}$ In severe cases of OHSS prophylactic anticoagulation should be used. In presence of thromboembolism therapeutic anticoagulation is indicated. ${ }^{[10,13]}$

\section{Conclusion}

In conclusion, OHSS should be considered in any women presenting with history of serous effusions and ovulation induction. The syndrome can range from mild symptoms to life-threatening pericardial effusions and myocardial ischemia. Prompt recognition and initiation of fluid therapy can reduce morbidity and prevent mortality.

\section{Reference}

1. Smits G, Olatunbosun O, Delbaere A, Pierson R, Vassart G, Costagliola $S$. Ovarian hyperstimulation syndrome due to a mutation in the follicle-stimulating hormone receptor. $\mathrm{N}$ Engl $\mathrm{J}$ Med 2003;349:760-6.

2. Kaiser UB. The pathogenesis of the ovarian hyperstimulation syndrome. N Engl J Med 2003;349:729-32.

3. Vasseur C, Rodien P, Beau I, Desroches A, Gerard C, de poncheville L, et al. A chorionic gonadotropin-sensitive mutation in the follicle-stimulating hormone receptor as a cause of familial gestational spontaneous ovarian hyperstimulation syndrome. $\mathrm{N}$ Engl J Med 2003;349:753-9.

4. Navot D. Severe ovarian hyperstimulation syndrome. In: Gardner DK, editor. Textbook of assisted reproductive techniques: Laboratory and clinical perspectives. $1^{\text {st }}$ ed. Martin Dunitz: London; 2001. p. 645-54.

5. Geva E, Jaffe RB. Role of vascular endothelial growth factor in ovarian physiology and pathology. Fertil Steril 2000;74:429-38.

6. Levin ER, Rosen GF, Cassidenti DL, Yee B, Meldrum D, Wisot A, et al. Role of vascular endothelial cell growth factor in ovarian hyperstimulation syndrome. J Clin Invest 1998;102:1978-85.
7. Navot D, Margalioth EJ, Luafer N, Birkenfeld A, Relou A, Rosler A, et al. Direct and severity of the ovarian hyperstimulation syndrome. Fertil Steril 1987;48:57-61.

8. Brinsden PR, Wada I, Tan SL, Balen A, Jacobs HS. Diagnosis, prevention and management of ovarian hyperstimulation syndrome. Br J Obstet Gynaecol 1995;102:767-72.

9. Forman RG, Frydman R, Egan D, Ross C, Barlow DH. Severe ovarian hyperstimulation syndrome using agonists of gonadotropinreleasing hormone for in vitro fertilization: A European series and a proposal for prevention. Fertil Steril 1990;53:502-9.

10. Avecillas JF, Falcone T, Arroligo AC. Ovarian hyperstimulation syndrome. Crit Care Clin 2004;20:679-95.

11. van Wely $M$, Westergaard LG, Bossuyt PM, van der Veen $F$. Effectiveness of human menopausal gonadotropin versus recombinant follicle-stimulating hormone for controlled ovarian hyperstimulation in assisted reproductive cycles: A meta-analysis. Fertil Steril 2003;80:1086-93.

12. Felberbaum RE, Diedrich K. Gonadotrophin releasing hormone antagonists: Will they replace the agonists? Reprod Biomed Online 2003;6:43-53.

13. Practice Committee of the American Society for Reproductive Medicine. Ovarian hyperstimulation syndrome. Fertil Steril 2004;82:S81-6.

14. Enskog A, Heriksson M, Unander M, Nilsson L, Brannstrom M. Prospective study of the clinical and laboratory parameters of patients in whom ovarian hyperstimulation syndrome developed during controlled ovarian hyperstimulation for in vitro fertilization. Fertil Steril 1999;71:808-14.

15. Delvigne A, Rozenberg S. Epidemiology and prevention of ovarian hyperstimulation syndrome (OHSS): A review. Hum Reprod Update 2002;8:559-77.

16. Semba S, Moriya T, Youssef EM, Sasano H. An autopsy case of ovarian hyperstimulation syndrome with massive pulmonary edema and pleural effusion. Pathol Int 2000;50:549-52.

17. Schenker JG, Weinstein D. Ovarian hyperstimulation syndrome: A current survey. Fertil Steril 1978;30:255-68.

18. Shanbhag S, Bhattacharya S. Current management of ovarian hyperstimulation syndrome. Hosp Med 2002;63:528-32.

19. Balasch J, Fabregues F, Arroyo V, Jimenez W, Creus M, Vanrell JA. Treatment of severe ovarian hyperstimulation syndrome by a conservative medical approach. Acta Obstet Gynecol Scand 1996;75:662-7.

20. Fabregues F, Balasch J, Gines P, Manau D, Jimenez W, Arroyo $\mathrm{V}$, et al. Ascites and liver test abnormalities during severe ovarian hyperstimulation syndrome. Am J Gastroenterol 1999;94:994-9.

21. Abramov Y, Naparstek Y, Elchalal U, Lewin A, Schechter E, Schenker JG. Plasma immunoglobulins in patients with severe ovarian hyperstimulation syndrome. Fertil Steril 1999;71:102-5. 
22. Tavmergen E, Ozcakir HT, Levi R, Adakan S, Ulukus M, Terek MC. Bilateral jugular venous thromboembolism and pulmonary emboli in a patient with severe ovarian hyperstimulation syndrome. J Obstet Gynaecol Res 2001;27:217-20.

23. Stewart JA, Hamilton PJ, Murdoch AP. Thromboembolic disease associated with ovarian stimulation and assisted conception techniques. Hum Reprod 1997;12:2167-73.

24. Heinig J, Behre HM, Klockenbusch W. Occlusion of the ulnar artery in a patient with severe ovarian hyperstimulation syndrome. Eur J Obstet Gynecol Reprod Biol 2001;96:126-7.

25. Abramov Y, Elchalal U, Schenker JG. Febrile morbidity in severe and critical ovarian hyperstimulation syndrome: A multicentre study. Hum Reprod 1998;13:3128-31.

26. Rabau E, David A, Serr DM, Mashiach S, Lunenfeld B. Human menopausal gonadotrophins for anovulation and sterility. Am J Obstet Gynecol 1967;98:92-8.

27. Navot D, Bergh PA, Laufer N. Ovarian hyperstimulation syndrome in novel reproductive technologies: Prevention and treatment. Fertil Steril 1992;58:249- 61.

28. Mathur RS, Akande AV, Keay SD, Hunt LP, Jenkins JM. Distinction between early and late ovarian hyperstimulation syndrome. Fertil
Steril 2000;73:901-7.

29. Practice committee of the American Society for Reproductive Medicine. Ovarian hyperstimulation syndrome. Fertil Steril 2003;80:1309-14.

30. Whelan JG $3^{\text {rd }}$, Vlahos NF. The ovarian hyperstimulation syndrome. Fertil Steril 2000;73:883-96.

31. Shanbhag S, Bhattacharya S. Current management of ovarian hyperstimulation syndrome. Hosp Med 2002;63:528-32.

32. Borenstein R, Elhalah U, Lunenfeld B, Schwartz ZS. Severe ovarian hyperstimulation syndrome: A reevaluated therapeutic approach. Fertil Steril 1989;51:791-5.

33. Zosmer A, Katz Z, Lancet M, Konichezky S, Schwartz-Shoham $Z$. Adult respiratory distress syndrome complicating ovarian hyperstimulation syndrome. Fertil Steril 1987;47:524-6.

34. Ware LB, Matthay MA. The acute respiratory distress syndrome. N Engl J Med 2000;342:1334-49.

35. Budev MM, Arroliga AC, Falcone T. Ovarian hyperstimulation syndrome. Crit Care Med 2005;33:S301-6.

Source of Support: Nil, Conflict of Interest: None declared.

\section{Author Help: Choosing an appropriate category of article for faster publication}

The manuscript system (w w w.journalonw eb.com) allow s the authors to check a likely publication date for a new ly submitted article. Based on number of articles in review, number of accepted articles and acceptance rate, the system estimates the likely publication date for an article submitted on a given date.

If there are too many articles in a category e.g., case report, a newly submitted case report if accepted may have to wait for a long period before publication. Hence, the author can check other categories e.g. letter to editor or images, for such paper and submit to another category of articles. 